\title{
Simultaneous Determination of Thermal Conductivity and Specific Heat for Refractory Materials
}

\author{
Kai $\mathrm{HO}^{\dagger}$ and Robert D. Pehlke \\ Department of Materials Science and Engineering, University of Michigan, \\ Ann Arbor, Michigan 48109-2136
}

\begin{abstract}
The present work describes a transient method for determining thermal properties of refractory materials. Specific to this technique are (1) the experimental measurement of heat flux on the heated surface and (2) a method of data analysis based on the numerical techniques of nonlinear least squares and finite difference. Several mathematical formulations are proposed to minimize computing time requirements. Silica molding sand is used in two examples to illustrate the applicability of the technique to refractory materials. Owing to the measurement of heat flux on the heated surface, both thermal conductivity $k$ and volumetric specific heat $C$ can be determined if their temperature dependencies are not considered. In the case when temperature-dependent thermal properties are considered, it is found that the inherent accuracy of the experiment does not allow the determination of both temperature-variable $k$ and $C$ at the same time. It is shown that the present method has advantages in the ease and directness of the required experiment and in greater computational efficiency. Thermal properties are reported for a bonded silica and an unbonded silica sand. [Key words: thermal conductivity, refractories, modeling, specific heat, thermal properties.]
\end{abstract}

\section{Introduction}

$\mathbf{I}^{\mathrm{N}}$ N THE thermal analyses of metallurgical processes, thermal properties of refractory materials are often required. Examples include molding sand in static-mold metal castings, linings in metallurgical furnaces, and mold powder in the continuous casting of steel. ${ }^{1}$ As a result, a rapid method for the determination of thermal properties is useful. In the following discussions, thermal properties refer to the quantities $k, C$, and $a$, where $k$ is the thermal conductivity and $C$ is the volumetric specific heat. The thermal diffusivity $a$ is a derived quantity given by $a=k / C$. Refractory materials are generally characterized by (1) a low thermal conductivity compared to metals and (2) a noticeable variation of thermal properties with temperature due to the large temperature differentials usually involved.

The present work is concerned with a comparison of mathematical techniques for computing thermal properties from a heating experiment. Only experiments involving a sample with a heated surface and a resulting temperature gradient (not calorimetric methods) are considered. From a mathematical point of view, these methods can be classified according to (1) truly steady-state, ${ }^{2-5}$ (2) quasi-steady-state, ${ }^{6-9}$ and (3) transient ${ }^{10-19}$ methods.

\section{(1) Truly Steady-State Methods}

These methods, suitable only for the measurement of $k$ but not $C$, depend on the measurement of the heat flux $q$ and the

\section{R. Bradt - contributing editor} Manuscript No. 198539. Received March 21, 1989; approved April 2, 1990.
'Deceased. temperature drop $\Delta T$ through a sample of thickness $\Delta x$ under steady-state conditions. For one-dimensional rectangular geometries, the thermal conductivity is given by the ratio of the heat flux $q$ to the temperature gradient $\Delta T / \Delta x$.

A steady-state method employing an absolute measurement of the magnitude of the heat flux has been published as an ASTM standard. ${ }^{2}$ In this method, refractory samples in the form of slabs are heated from above by an electrical heat source and cooled from below by a water-cooled heat sink. From the temperature rise of the cooling water through a central heat sink, the heat flux through a central sample can be calculated. On the other hand, the measurement of heat flux can also be carried out by comparative means ${ }^{3}$ using standard refractory samples of known thermal conductivity in series with the unknown sample. Refractory materials suitable for a standard in thermal conductivity include alumina, titanium carbide, and fosterite. ${ }^{3}$

\section{(2) Quasi-Steady-State Methods}

In contrast to the truly steady-state methods, quasi-steadystate methods utilize nonsteady temperature measurements (changing with time), but the mathematical analysis is based on a quasi-steady-state solution to the transient heat equation at sufficiently long times, such that temperature varies linearly with the logarithm of time ${ }^{6-8}$ or the time scale itself. ${ }^{9}$ Examples of the first type include the work of Roshan et al. ${ }^{6}$ and Hartley et al.,",8 who applied the method of "thermal probe" to the measurement of thermal conductivity in molding sands.

\section{(3) Transient Methods}

Transient methods derive their analyses from the transient solution of the Fourier equation of heat conduction. For these methods, one of the quantities $k, C$, or $a$ may be determined from each experiment when temperature measurements alone are used. If the surface heat flux, as well as temperatures, are measured, two of the quantities $k, C$, or $a$ may be determined in a single experiment. It should also be noted that only the volumetric specific heat $C$, but not the specific heat based on unit mass $c$, can be derived by a transient heating experiment. A separate gravimetric experiment to measure the density $\rho$ is necessary in order to calculate the specific heat based on unit mass $c=C / \rho$ from a knowledge of $c$.

In the present work, a technique which allows the measurement of surface heat flux in a transient heating experiment is developed. The accuracy of this technique is such that only temperature-averaged $k$ and $C$ can be determined simultaneously from a single experiment, or if a temperaturedependent $C$ available from the literature is used as a known quantity in the data analysis, it is found possible to derive a temperature-dependent $k$ in the form of a polynomial.

\section{Experimental Procedure}

Figure 1 shows the design of the present experiment. It consists of a central mold cavity in which molten metal serves as the heat source. Using silica sand as the molding material, two 




Fig. 1. Schematic diagram of the experimental arrangement.

heating experiments were performed with the silica sand prepared in a different condition with respect to binder content.

In the first experiment, the molding material consisted of silica sand ${ }^{\ddagger}$ with $4 \%$ western bentonite and $2 \%$ water by weight and was rammed around a cylindrical mold cavity $8.26 \mathrm{~cm}$ in radius and $16.5 \mathrm{~cm}$ in height. Both the top and bottom portion of the mold cavity were thermally insulated with Kaowool ${ }^{\S}$ boards (an insulating ceramic fiber), which extended laterally into the molding medium. The mold assembly with the ceramic fiber boards was dried in an oven at $350^{\circ} \mathrm{C}$ for more than $24 \mathrm{~h}$ before the experiment. A small opening was drilled through the Kaowool board located at the top of the mold in order to allow the entrance of liquid metal into the mold cavity during pouring. Straight pieces of type-k thermocouples, protected in small-diameter fused silica tubing with the junctions at the midheight, were inserted vertically in the mold at various radial distances (see Fig. 2). The transient temperatures recorded by these thermocouples were transmitted to a digital data logging system during the progress of the experiment. A metal with a relatively low melting point, commercially pure aluminum with a melting point of $660^{\circ} \mathrm{C}$, was chosen as the cast metal which was poured with a high degree of superheat (pouring temperature $1200^{\circ} \mathrm{C}$ ). Owing to the thorough mixing as a result of liquid convection within the highly superheated melt, the thermal gradient in the melt was sufficiently small to ailew the calculation of heat flux into the mold by measuring the cooiing rate of the bulk liquid. The entire collection of temperature tata, for thermocouples placed in the molding sand and molten metal, was recorded digitally on cassette tape and then transferred to a mainframe computer for analysis.

In order to illustrate the effect of binder content on the thermal conductivity of a granulated material, the second ex-

\footnotetext{
${ }^{\ddagger}$ AFS-85 mesh Wedron silica sand, Wedron Silica Co., Wedron, IL. Thermal Ceramics, Augusta, GA.
}

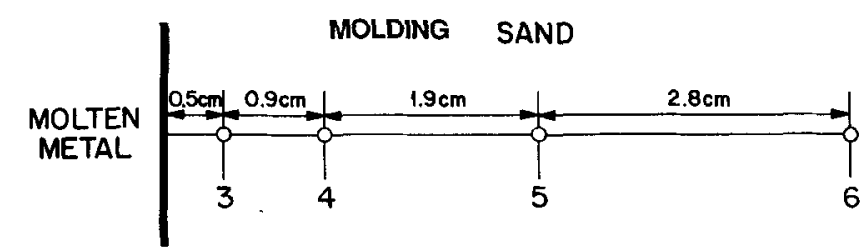

Fig. 2. Positions of the thermocouples in the sand mold for the first experiment. periment utilized unbonded silica sand" as the molding medium. The use of unbonded silica sand has practical applications in the vacuum molding and the lost foam processes of casting metals. Two major modifications were adopted with respect to the first experiment. First, a cylindrical can made of thin sheet steel was inserted into the central mold cavity to provide the necessary structural support against the unbonded sand. Second, since the measurement of heat flux was not planned in the second experiment, thermocouples were not needed in the mold cavity and the thermal properties of the cast metal need not be known. Consequently, a remelted brass was used as the cast metal. The dimensions of the apparatus were comparable to those of the first experiment. As a heat source, the use of brass provides an approximate thermal arrest during solidification at a sufficiently high temperature, approximates a step increase in temperature after pouring, and hence represents a favorable heating condition for the transient determination of thermal properties. ${ }^{15}$ In comparison, the use of an electric heat source is not suitable for the intended numerical method of analysis, because (1) the weaker heat flux of an imbedded electric heat source, compared to molten metals, results in a much longer heating time and deeper thermal penetration into the mold for an appreciable increase in surface temperature, so that the assumption of one-dimensional heat flow is no longer valid, and (2) the transient sensitivity coefficients, ${ }^{15}$ which represent a measure of the degree of accuracy attainable during the computation of thermal properties from temperature responses, are much diminished if the heating at the boundary is imparted by a constant heat flux (such as that arising from the passage of a steady electric current), as compared to the case of a step increase in the surface temperature approximated by the use of molten metal as a heat source.

\section{Mathematical Analysis}

The present work has adopted the technique of nonlinear least squares and the method of finite difference for the analysis of experimental data. Given some initial estimate of the thermal properties $k$ and $C$, the finite-difference method calculates the temperatures in the refractory mold as a function of position and time. For generality, these thermal properties may be regarded as temperature dependent. By a systematic variation of $k$ and $C$, the method of nonlinear least squares proceeds to minimize the function

$$
F(k, C)=\sum_{n=1}^{N}\left(T_{n}-\Theta_{n}\right)^{2}
$$

which represents a quantitative measure of the degree of agreement between the calculated and experimentally measured temperature. Here $T_{n}$ is the calculated temperature and $\Theta_{n}$ the measured temperature of the $n$th measurement. The subacript $n$ is incremented with time among the thermocouples as the diroital data logging system selected the thermocouples in the sand nold in a cyclic manner. The quantity $N$ denotes the total nunber of thermocouple readings in the entire experiment.

A number of mathematical techniques exist for minimizing Eq. (1). In the original work of Beck, ${ }^{15}$ a basic technique, involving a local Taylor series expansion and the variation of one independent variable ( $k$ or $C$ ) at a time, was adopted. Kubo et al. ${ }^{19}$ used a minimizing tecisnique as proposed by Powell. ${ }^{20}$ The present work has attempted two alternative methods: (1) the basic technique (see App ondix A) and (2) the use of an existing FORTRAN subroutins ZXSSO from the IMSL library ${ }^{21}$ available for the authors' access on a computer. ${ }^{\dagger \dagger}$ In the latter scheme, the evaluation on the function $F$

\footnotetext{
'Wedron 420 Grade silica sand.

t+Amdahl 470/V8, Amdahl Computer, Sunnyvale, CA.
} 
itself is accomplished by a finite-difference model coded as a subroutine which is passed as an actual parameter to the calling subroutine ZXSSQ. This finite-difference model allows the use of an unevenly spaced grid placed in such a way that (1) each thermocouple position is precisely matched with a nodal position and (2) a finer grid spacing is employed near the heated surface where the temperature change is more rapid in order to improve accuracy. A mathematical description of the finite-difference model is included in Appendix B.

In addition to measurements of temperatures in the molding sand, the present work also attempted to measure the heat flux from the molten metal into the surface of the mold. A measurement of heat flux is necessary because, as shown by Beck, ${ }^{13}$ it is impossible to determine both $k$ and $C$ in one experiment if temperature data alone are used, as $k$ and $C$ would then become linearly dependent during the mathematical process of nonlinear least squares. The measurement of heat flux on the heated surface removes this linear dependency. In the present experiments, the heating conditions provide an approximately one-dimensional heat flow along the outward radial direction. Furthermore, in the first experiment, the temperature in the bulk of the highly superheated aluminum was essentially uniform. It follows that the radial heat flux into the surface of the mold could be calculated from the rate of change of temperature of the metal. In the actual numerical computations, the measured temperature $T$ in the liquid metal was smoothed by a cubic spline/least squares procedure first, before the differentiation process was carried out on the spline curves in order to obtain the cooling rate $\mathrm{d} T / \mathrm{d} t$. The thermophysical properties of liquid aluminum were taken from Ref. 22 . The approximate mean values of $2340 \mathrm{~kg} \cdot \mathrm{m}^{-3}$ and $1086 \mathrm{~J} \cdot \mathrm{kg}^{-1} \cdot \mathrm{K}^{-1}$ were used for the density and specific heat of liquid aluminum, respectively.

\section{Results}

\section{(1) A First Experiment on Bonded Silica Sand}

Figure 3 shows the measured temperatures from two thermocouples located in the molten metal as a function of time. Except during an initial period of approximately $3 \mathrm{~min}$, the temperatures measured by these two thermocouples remain indistinguishable from each other in the graph. This result therefore verifies the assumption that the temperature gradient in the molten metal is negligible. Consequently, the time-dependent heat flux into the mold may be evaluated from

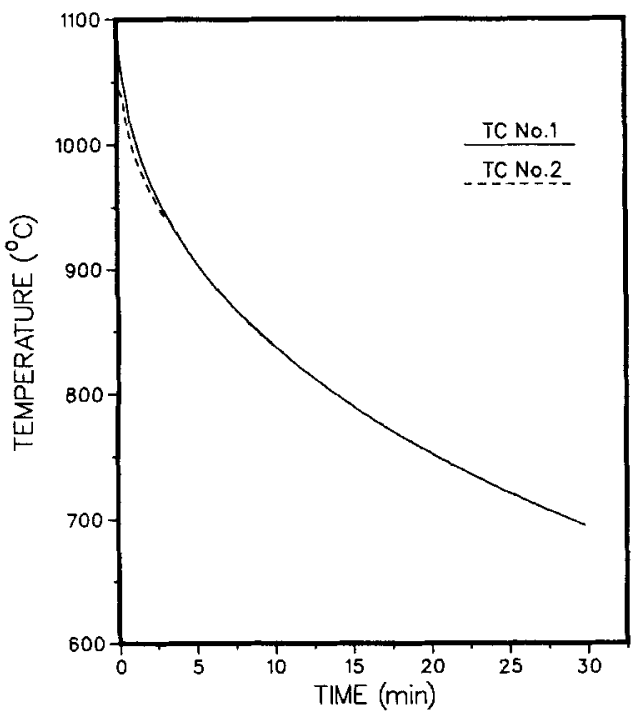

Fig. 3. Measured temperatures in liquid aluminum as a function of time in the first experiment. Refer to Fig. 1 for the locations of the thermocouples. the cooling rate of the molten metal. Two analyses were carried out on the experimental data:

Analysis I (temperature-independent $k$ and $C$ )-In this analysis, the heat flux calculated from the cooling rate of the liquid metal is used as a Neumann boundary condition on the heated surface of the mold, and the temperature as recorded by thermocouple 6 (see Fig. 2 ) is used as a Dirichlet boundary condition on the unheated circumference. The corresponding relationship between the variable $n$ in Eq. (1) and the thermocouple designation is shown in Table I. Starting with an initial estimate of $k=1.0 \mathrm{~W} \cdot \mathrm{m}^{-1} \cdot \mathrm{K}^{-1}$ and $C=2.0 \mathrm{MJ} \cdot \mathrm{m}^{-3} \cdot \mathrm{k}^{-1}$, the process of nonlinear least squares converges after 12 iterations (with a total of 36 evaluations of the finite-difference calculation), giving, to three significant figures, the values of $k$ and $C$ shown in Table II. In addition, an rms value representing the deviation between calculated temperatures and the experimental data, defined by the expression

$$
\left[\frac{1}{N} \sum_{n=1}^{N}\left(T_{n}-\Theta_{n}\right)^{2}\right]^{1 / 2}
$$

was evaluated during the finite-difference computation as an indication of the agreement between calculation and experiment. In the present case, the rms value after convergence is found to be $22.1 \mathrm{~K}$. A graphical representation of the agreement between the calculated and measured temperatures at various thermocouples is shown in Fig. 4.

Analysis II (temperature-dependent $k$ )-The above analysis shows how temperature-independent $k$ and $C$ could be derived from the present experiment. However, an attempt to extend analysis I to the simultaneous determination of temperaturedependent $k$ and $C$ does not meet with success for the following reasons: (a) The measured surface heat flux has only a limited accuracy because of neglecting longitudinal heat loss into the insulating fiber material. Owing to the relatively high insulating properties of silica sand, its thermal conductivity is not sufficiently large, compared to that of the insulating fiber material, for the assumption of one-dimensional radial heat flow to be highly accurate. (b) Temperature-independent density and specific heat are used for liquid aluminum. (c) As discussed earlier, heating conditions of a transient experiment have a strong influence on the accuracy of the thermal properties to be determined. The ideal heating condition has not been precisely achieved in the present experiment.

Although the present experiment does not permit a simultaneous determination of two temperature-variable properties

Table I. Thermocouple Increment Index $\boldsymbol{n}$ for Analyses $I$ and II of the First Experiment

\begin{tabular}{|c|c|}
\hline$n$ (Eq. (1)) & Thermocouple designation \\
\hline \multicolumn{2}{|c|}{ Analysis I* } \\
\hline 1 & 3 \\
\hline 2 & 4 \\
\hline 3 & 5 \\
\hline 4 & 3 \\
\hline 5 & 4 \\
\hline 6 & 5 \\
\hline - & . \\
\hline - & $\cdot$ \\
\hline - & . \\
\hline
\end{tabular}

Analysis II ${ }^{\dagger}$

$\begin{array}{ll}1 & 4 \\ 2 & 5 \\ 3 & 4 \\ 4 & 5\end{array}$

\footnotetext{
*The heat flux measured by the cooling rate of the molten metal is used as Neumann boundary condition on the heated surface.

Neumann boundary condition on the heated surface.
Temperatures measured by TC3 are used as a Dirichlet boundary condition on the heated surface.
} 
Table II. Results of Analyses I and II on the Thermal Properties for the First Experiment: Dried Silica Sand (AFS-85 Mesh Wedron) with 4\% Binder



$k$ and $C$, it is found possible to determine one temperaturedependent property if the other property is treated as a known quantity in the numerical analysis. Furthermore, in order to avoid the inaccuracy introduced by the use of an inaccurate surface heat flux, the present analysis is carried out using thermocouple 3 as a Dirichlet boundary condition in place of the heat flux boundary condition. Consideration of the heat transfer mechanisms shows that the specific heat of a molding sand, when compared to the thermal conductivity, is much less dependent of the packing, grain size, binder content, etc. Consequently, the present analysis utilizes as a known quantity ${ }^{23}$ the volumetric specific heat of silica sand in the form

$$
C=1.590 \times 10^{6}+4.038 \times 10^{2} T-4.896 \times 10^{10} / T^{2}
$$

where $C=\rho c$ is in units of $\mathrm{J} \cdot \mathrm{m}^{-3} \cdot \mathrm{K}^{-1}$ and $T$ is in kelvins. In this equation, a term involving the reciprocal expression $1 / T^{2}$ is used instead of a $T^{2}$ term in accordance with the usual practice of describing the variation of specific heat with temperature. Using this functional relationship for the volumetric specific heat, the analysis proceeds to determine a temperature-dependent thermal conductivity in the form

$$
k=a_{0}=a_{1} T+a_{2} T^{2}
$$

where the temperature is expressed in kelvins. For numerical efficiencies, it is found that instead of minimizing $F$ in Eq. (1) with respect to the coefficients $a_{0}, \ldots, a_{2}$ in Eq. (4), a more rapid convergence can be achieved in the process of nonlinear least squares by minimizing $F$ with respect to three thermal conductivities $k_{1}, \ldots, k_{3}$ at three reference temperatures $T_{1}, \ldots, T_{3}$. The coefficients in Eq. (4) can then be determined by solving a system of linear equations when these conductivities and temperatures are substituted into Eq. (4). This treatment can be extended to the use of higher-order polynominal or other functional relationships for $k$ in a straightforward manner. In the case of a discontinuity in $k$ with temperature due to phase transformation, special treatments such as the use of piecewise polynomials can be used.

Using an initial estimate of $k_{1}=k_{2}=k_{3}=0.7 \mathrm{~W} \cdot \mathrm{m}^{-1}$. $\mathrm{K}^{-1}$, the process of nonlinear least squares converges after 5 iterations (a total of 20 evaluations of the finite-difference calculation) for the variables $k_{1}, \ldots, k_{3}$ to agree within three significant figures between successive iterations. The calculated and measured temperatures at thermocouples 4 and 5, having an overall $\mathrm{rms}$ deviation of $3.15 \mathrm{~K}$, are graphically displayed in Fig. 5. The coefficients of the resulting seconddegree polynomial for thermal conductivity are presented in Table II, and the variation of thermal conductivity with temperature is graphically displayed in Fig. 6 . The resulting polynomial for $k$ is applicable up to a certain limit in temperature, which may be roughly taken as the maximum temperature attained in the Dirichlet boundary condition of the heated end (i.e., thermocouple 3 in the present analysis). Included in the same graph are discrete data points from Atterton ${ }^{4}$ using a steady-state technique, and Hartley and Patterson ${ }^{8}$ using a

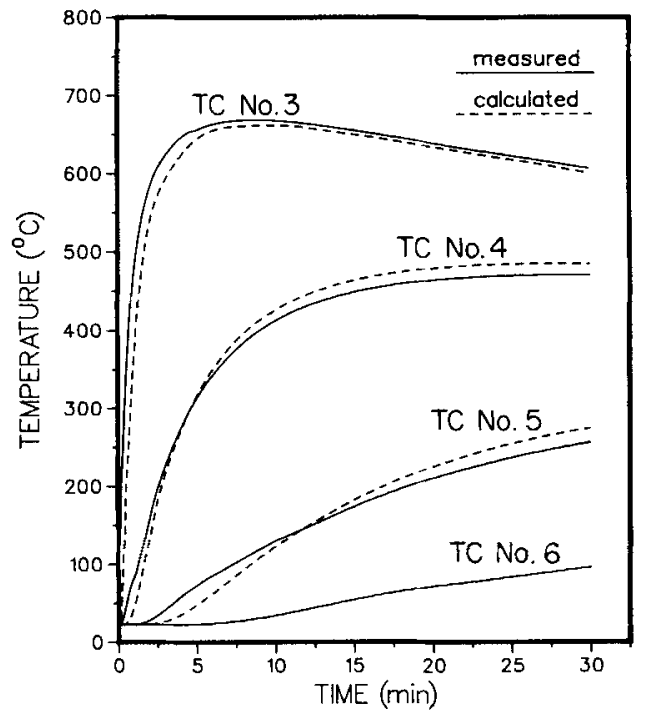

Fig. 4. Calculated and measured temperatures for analysis I of the first experiment - a heat flux boundary condition at the metal-mold interface and a Dirichlet boundary condition at thermocouple 6 .

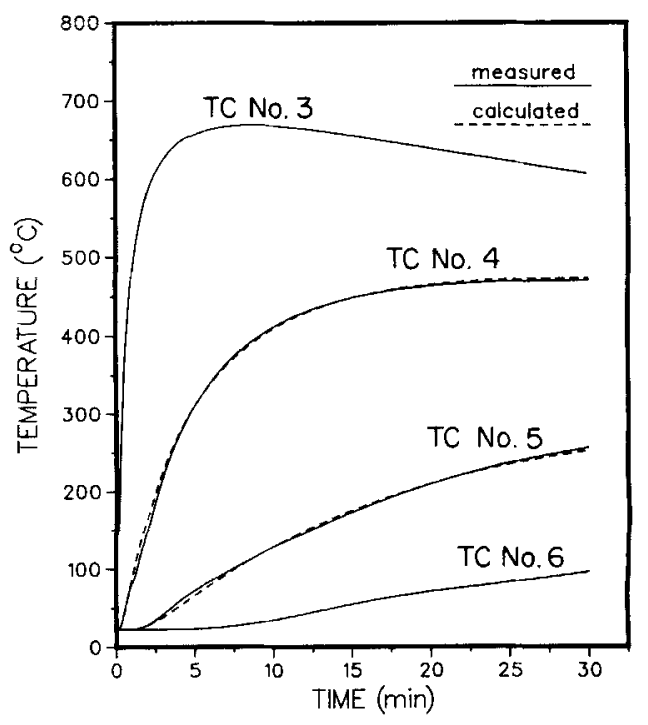

Fig. 5. Calculated and measured temperatures for analysis II of the first experiment-temperatures measured at thermocouples 3 and 6 are used as Dirichlet boundary conditions. 


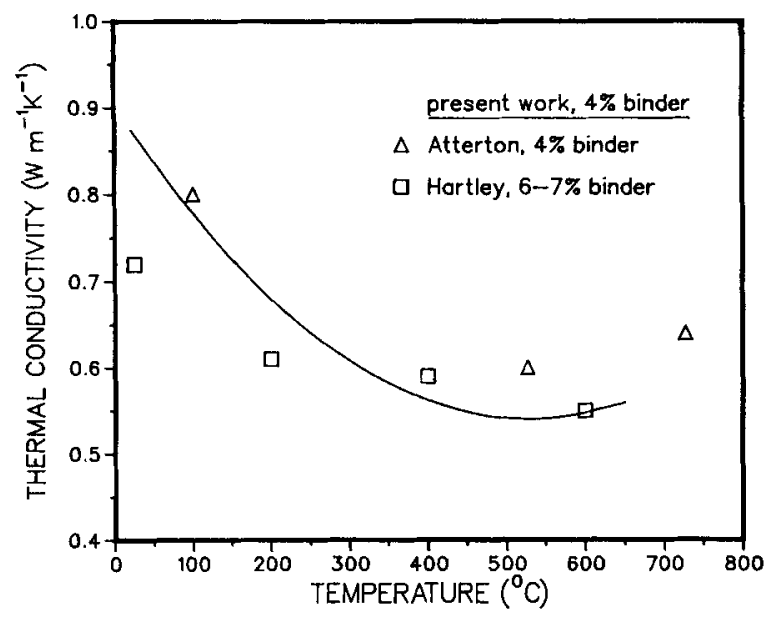

Fig. 6. Computed temperature variation of thermal conductivity for the first experiment - a dried silica sand (AFS85 mesh Wedron) with $4 \%$ binder. Included in the same graph are the results from Atterton, ${ }^{4}$ and Hartley and Patterson.

quasi-steady-state technique. Beyond approximately $500^{\circ} \mathrm{C}$, the increase in thermal conductivity with a further increase in temperature can be ascribed to the onset of the importance of intergranular radiation at higher temperatures. In view of the possible differences in grain size distribution and degree of packing, etc., as well as the systematic errors introduced by each particular method, the agreement should be regarded as satisfactory.

A comparison of the constant thermal conductivity $k$ derived under analysis I (Table II) and the temperaturedependent $k$ (Fig. 6) shows that the former value lies between the maximum and minimum values of $k$ displayed in Fig. 6. This result therefore shows that analysis I produces an acceptable value for the thermal conductivity in an average sense. The error introduced by the use of constant thermal properties in the former case results in a deviation in temperature which is greater for analysis I (Fig. 4) compared to analysis II (Fig. 5).

Two slightly different versions of the described computer program were written to account for the Neumann (heat flux) boundary condition on the heated surface in analysis $I$ and the Dirichlet (temperature specified) boundary condition in analysis II. The actual computations required $5.8 \mathrm{~min}$ for analysis I on our computer, ${ }^{\dagger \dagger}$ and 3 min for analysis II.

\section{(2) A Second Experiment on Unbonded Silica Sand}

Figure 7 shows the computed temperature dependence of thermal conductivity for an unbonded silica sand, using

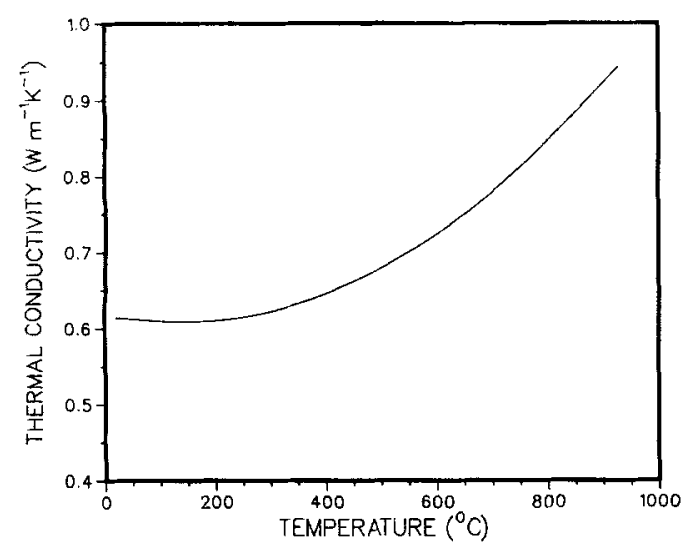

Fig. 7. Computed temperature variation of thermal conductivity for the second experiment - an unbonded silica sand (Wedron $420 \mathrm{Grade}$ ). analysis II of the previous experiment. The corresponding polynomial for $k$ is given by

$$
k=0.6928-4.236 \times 10^{-4} T+5.267 \times 10^{-7} T^{2}
$$

Compared to Fig. 6, which shows the temperature-dependent $k$ in the case of a bonded sand ( $4 \%$ binder), it can be seen that the conductivity for unbonded sand is much lower than that of bonded sand at lower temperatures. This behavior can be explained on the basis that at lower temperatures, thermal conduction along the sand grain is the main mode of heat transfer and the lack of binder in an unbonded sand results in a higher heat transfer resistance at the interface between sand grains. At higher temperatures, on the other hand, thermal radiation in the void spaces between sand grains, as well as along the interior of the sand grains, becomes more important and these modes of heat transfer are quite independent of the presence of a binder. However, since silica sands of different grain sizes have been used for the two experiments, a direct comparison of the absolute magnitude of thermal conductivity at higher temperatures cannot be made.

\section{Conclusions}

A straightforward experimental technique for the measurement of heat flux into a heated surface has been developed. The accuracy of the heat flux measurement is such that temperature-independent (but not temperature-variable) thermal conductivity and specific heat can be determined simultaneously in a single experiment.

A mathematical method for computing the thermal properties of refractory materials in a single transient heating experiment, using the techniques of nonlinear least squares and finite-difference techniques, is described. The approach is demonstrated using measurement of a boundary heat flux (Neumann condition) to determine temperature-independent $k$ and $C$, and using a defined higher temperature boundary (Dirichlet condition) to determine a temperature-dependent $k$. Advantages over other techniques are noted.

The thermal conductivities of a bonded and an unbonded silica sand have been determined as a function of temperature over the temperature range from $25^{\circ}$ to $660^{\circ} \mathrm{C}$ and from $25^{\circ}$ to $900^{\circ} \mathrm{C}$, respectively. Average values for $k$ and $C$ of a bonded sand over the temperature range from $25^{\circ}$ to $660^{\circ} \mathrm{C}$ are also reported.

\section{APPENDIX A}

The present treatment shows how the basic technique for minimizing a sum of squares can be used to derive a temperature-dependent thermal conductivity $k$ from a transient heating experiment. Although more elaborate techniques exist, a discussion of the basic technique nevertheless provides some insight into the nature of the minimization process. For convenience, let the temperature variation of $k$ be represented by

$$
k=a_{0}+a_{1} T+a_{2} T^{2}
$$

Other functional relationships between $k$ and $T$ could be used, but a function which is linear with respect to the unknown coefficients (denoted by $a_{0}, \ldots, a_{2}$ above) is more convenient because it avoids the necessity of solving a nonlinear system of equations during a determination of unknown coefficients from known values of $k$ and $T$.

The nonlinear least-squares method seeks to minimize the function

$$
F(k)=\sum_{n=1}^{N}\left(T_{n}-\Theta_{n}\right)^{2}
$$

by adjusting the parameters $a_{0}, \ldots, a_{2}$ in Eq. (A-1). In Eq. $(A-2), \Theta_{n}$ represents the $n$th measured temperature recorded by a multichannel scanning device as illustrated in 
Table II, and $T_{n}$ represents the calculated temperature using finite difference at the location and time designated by $n$. For computational efficiencies, it is more desirable to minimize $F$ with respect to three thermal conductivities $k_{1}, \ldots, k_{3}$ at three reference temperatures $T_{1}, \ldots, T_{3}$. The corresponding values of $a_{0}, \ldots, a_{2}$ can then be determined by solving Eq. (A-1). Conditions for minimizing the function $F$ in Eq. $(A-2)$ are given by

$$
\frac{\partial F}{\partial k_{i}}=0 \quad(i=1, \ldots, 3)
$$

This minimization condition can be solved numerically as follows. Let $k_{1}, \ldots, k_{3}$ be a sequence of iterated values of the thermal conductivities at iteration stage $m$ where $m=$ $1,2,3, \ldots$, etc. At stage $m$, the corrections to these thermal conductivities are found using a Taylor series expansion

$$
\begin{aligned}
T_{n} \simeq & T_{n}\left(k_{1}^{m}, k_{2}^{m}, k_{3}^{m}\right)+\frac{\partial T_{n}}{\partial k_{1}} \Delta k_{1}+\frac{\partial T_{n}}{\partial k_{2}} \Delta k_{2} \\
& +\frac{\partial T_{n}}{\partial k_{3}} \Delta k_{3}
\end{aligned}
$$

where

$$
\begin{aligned}
& n=1, \ldots, N \\
& \Delta k_{i}=k_{i}-k_{i}^{m} \\
& i=1, \ldots, 3
\end{aligned}
$$

Substitution of Eq. (A-4) into Eq. (A-3) gives

$$
\begin{aligned}
& \sum_{n=1}^{N}\left[\left(T_{n}\left(k_{1}^{m}, k_{2}^{m}, k_{3}^{m}\right)+\frac{\partial T_{n}}{\partial k_{1}} \Delta k_{1}\right.\right. \\
& \left.\left.\quad+\frac{\partial T_{n}}{\partial k_{2}} \Delta k_{2}+\frac{\partial T_{n}}{\partial k_{3}} \Delta k_{3}\right) \frac{\partial T_{n}}{\partial k_{i}}\right]=0 \\
& (i=1, \ldots, 3)
\end{aligned}
$$

In order to simplify notations, the inner product defined by

$$
(A, B)=\sum_{n=1}^{N} A_{n} B_{n}
$$

will be used. Equation (A-5) becomes, in matrix form

$$
\begin{aligned}
& {\left[\begin{array}{lll}
\left(\frac{\partial T}{\partial k_{1}}, \frac{\partial T}{\partial k_{1}}\right) & \left(\frac{\partial T}{\partial k_{1}}, \frac{\partial T}{\partial k_{2}}\right) & \left(\frac{\partial T}{\partial k_{1}}, \frac{\partial T}{\partial k_{3}}\right) \\
\left(\frac{\partial T}{\partial k_{2}}, \frac{\partial T}{\partial k_{1}}\right) & \left(\frac{\partial T}{\partial k_{2}}, \frac{\partial T}{\partial k_{2}}\right) & \left(\frac{\partial T}{\partial k_{2}}, \frac{\partial T}{\partial k_{3}}\right) \\
\left(\frac{\partial T}{\partial k_{3}}, \frac{\partial T}{\partial k_{1}}\right) & \left(\frac{\partial T}{\partial k_{3}}, \frac{\partial T}{\partial k_{2}}\right) & \left(\frac{\partial T}{\partial k_{3}}, \frac{\partial T}{\partial k_{3}}\right)
\end{array}\right]} \\
& {\left[\begin{array}{l}
\left(\frac{\partial T}{\partial k_{1}^{m}}, \Theta-T\left(k_{1}^{m}, k_{2}^{m}, k_{3}^{m}\right)\right) \\
\Delta k_{2}^{m} \\
\Delta k_{3}^{m}
\end{array}\right]=} \\
& {\left[\begin{array}{l}
\left(\frac{\partial T}{\partial k_{2}}, \Theta-T\left(k_{1}^{m}, k_{2}^{m}, k_{3}^{m}\right)\right) \\
\left(\frac{\partial T}{\partial k_{3}}, \Theta-T\left(k_{1}^{m}, k_{2}^{m}, k_{3}^{m}\right)\right)
\end{array}\right]}
\end{aligned}
$$

where the matrix on the left-hand side is symmetric because of the commutative property of inner products. Since the partial derivatives occurring in the inner products cannot be evaluated analytically, each element of the vector

$$
\frac{\partial T}{\partial k_{i}}=\left[\frac{\partial T_{1}}{\partial k_{i}} \frac{\partial T_{2}}{\partial k_{i}} \ldots \frac{\partial T_{N}}{\partial k_{i}}\right]
$$

is evaluated using the finite-difference method according to the expression (illustrated for the case $i=1$ )

$$
\frac{\partial T_{n}}{\partial k_{1}} \simeq \frac{T_{n}\left(k_{1}(1+\epsilon), k_{2}, k_{3}\right)-T_{n}\left(k_{1}, k_{2}, k_{3}\right)}{\epsilon k_{1}}
$$

where $\epsilon \ll 1$. The iteration process starts by some initial guess of $k_{1}^{1} \ldots k_{3}^{1}$. By evaluating the inner products and solving Eq. (A-7), the thermal conductivities in the next iteration are obtained by

$$
k_{i}^{2}=k_{i}^{1}+\Delta k_{i}^{1}
$$

where the superscript refers to the iteration stage. The iteration process can be repeated until some convergence criteria such as

$$
\left|\frac{\Delta k_{i}}{k_{i}}\right|<0.001 \quad(i=1, \ldots, 3)
$$

is reached.

In the initial stage of the present work, this algorithm has been coded as part of a main computer program (i.e., not a subroutine) and the numerical result was found to be identical to that produced by the IMSL ${ }^{21}$ subroutine ZXSSQ. Since it is more convenient to make changes to a computer program when the nonlinear least-squares algorithm is coded separately in the form of a subroutine, subsequent developments, including all results presented in this work, have been computed by the use of the ZXSSQ subroutine.

\section{APPENDIX B}

Consider the experimental arrangement as shown in Fig. 1. Since the flow of heat is approximately one-dimensional along the outward radial direction, the transient equation of heat conduction is given by

$$
\frac{1}{r} \frac{\partial}{\partial r}\left(r k \frac{\partial T}{\partial r}\right)=C \frac{\partial T}{\partial t}
$$

where $r$ is the radial distance and $t$ is the time. In this equation, the thermal conductivity $k$ occurs inside one of the differential operators. Special treatment in the finite-difference formulation is therefore required if the quantity $k$ varies with temperature. The present treatment starts by expanding the left-hand side of Eq. (B-1) in the form

$$
k \frac{\partial^{2} T}{\partial r^{2}}+\left[\frac{k}{r}+\frac{\partial k}{\partial T} \frac{\partial T}{\partial r}\right] \frac{\partial T}{\partial r}
$$

In order to maintain linearity in the final system of equations in the finite-difference method, a linearization technique is applied to expression (B-2), giving

$$
k^{*} \frac{\partial^{2} T}{\partial r^{2}}+\left[\frac{k^{*}}{r}+\left(\frac{\partial k}{\partial T}\right)^{*}\left(\frac{\partial T}{\partial r}\right)^{*}\right] \frac{\partial T}{\partial r}
$$

where the superscript ${ }^{*}$ denotes quantities which are evaluated at the old finite-difference time level. The linearity of the resulting system of equations can be illustrated by applying this equation to a general interior node $i$ with neighboring nodes $i-1$ and $i+1$ evenly spaced with spacing $\Delta r$ from the center node $i$. Using a Crank-Nicolson time stepping scheme, the derivative may be written as

$$
\begin{aligned}
& \frac{\partial^{2} T}{\partial r^{2}}=\frac{1}{2}\left[\frac{T_{i+1}-2 T_{\mathrm{i}}+T_{i-1}}{(\Delta r)^{2}}\right.\left.+\frac{T_{i+1}^{*}-2 T_{i}^{*}+T_{i-1}^{*}}{(\Delta r)^{2}}\right] \\
& \frac{\partial T}{\partial r}=\frac{1}{2}\left[\frac{T_{i+1}-T_{i-1}}{2 \Delta r}+\frac{T_{i+1}^{*}-T_{i-1}^{*}}{2 \Delta r}\right] \\
& \frac{\partial T}{\partial t}=\frac{T_{i}-T_{i}^{*}}{\Delta t}
\end{aligned}
$$


When these derivatives are substituted into expression (B-3), the linearity can readily be observed (i.e., unknown temperatures $T_{i-1}, T_{i}$, and $T_{i+1}$ at the new time level occur as a first power only). In a similar manner, finite-difference equations for nodes situated at the boundaries can be derived. In the case of a node with neighboring nodes unevenly spaced from it, the finite-difference equation utilizes three neighboring nodes. Mathematical derivations of the basic finite-difference equation are described in Ref. 24 . The resulting system of linear equations is solved by a banded matrix subroutine LEQT1B from the IMSL library. ${ }^{21}$

\section{References}

${ }^{1}$ H. J. Heine, "Refractories for Metalcasting-Part I," Foundry Manag. Technol, 113, 26-32 (1985).

${ }^{2}$ ASTM Designation C201-68, "Standard Test Method for Thermal Conductivity of Refractories"; pp. 181-87 in Annual Book of ASTM Standards, Part 17. American Society for Testing and Materials, Philadelphia, PA, 1981.

${ }^{3}$ V.V. Mirkovich, "Comparative Method and Choice of Standards for Thermal Conductivity Determinations," J. Am. Ceram. Soc., 48 [8] 387-91 (1965)

${ }^{4} \mathrm{D}$.V. Atterton, "The Apparent Thermal Conductivities of Moulding Materials at High Temperatures," J. Iron Steel Inst., 174, 201-11 (1953).

${ }^{5}$ D. H. Whit more and Q. F. Ingerson, "Apparent Thermal Conductivity of Molding Sands at Elevated Temperatures," Trans. Am. Foundrymen's Soc., 68, 49-57 (1960).

${ }^{6 H}$. M. Roshan, E. G. Ramachandran, A. Ramachadran, and M. R. Seshadri, "Thermal Properties of Resin Bonded Sand," Br. Foundryman, 68, $14-20(1975)$.

JJ. G. Hartley, D. Babcock, and J.T. Berry, "The Thermal Conductivity of Bentonite-Bonded Molding Sands," Trans. Am. Foundrymen's Soc., 89, 469-76 (1981).

${ }^{8}$ J. G. Hartley and J.A. L. Patterson, "The Influence of Temperature, Moisture Content and Binder Content on the Thermal Conductivity of Dried Bentonite-Bonded Zircon and Silica Sands," Trans. Am. Foundrymen's Soc., 91, 183-89 (1983).

${ }^{9}$ H. Saito, N. Seki, and S. Sakatsume, "An Investigation of Heat Transfer Coefficients of Some Molding Sands," Trans. Am. Foundrymen's Soc., 84,
243-48 (1976).

${ }^{10}$ R. Taylor and K.C. Mills, "Physical Properties of Casting Powders: Part 3, Thermal Conductivity of Casting Powders," Ironmaking Steelmaking, $15,187-94(1988)$

${ }^{11} \mathrm{H}$. J. Lee and R. Taylor, "Determination of Thermophysical Properties of Layered Composites by Flash Method"; pp. 423-34 in Thermal Conductivity 14. Edited by P. G. Klemens and T. K. Chu. Plenum Press, New York, 1976.

${ }^{12}$ M. Faucher, F. Cabannes, A. M. Anthony, B. Piriou, and J. Simonato, "Arc-Imaging Technique for Measuring High-Temperature Thermal Conductivity and Diffusivity of Refractory Oxides," J. Am. Ceram. Soc., 58 [9-10] 368-71 (1975).

${ }^{13}$ R.W. Ruddle and A.L. Mincher, "The Thermal Properties and Chilling Power of Some Non-Metallic Moulding Materials," J. Inst. Met., [Sept.] 4390 (1949).

${ }^{14}$ M. J. Kirt and R. D. Pehlke, "Determination of Material Thermal Properties Using Computer Techniques," AFS Cast Met. Res. J., 9, 117-21 (1973).

${ }^{15}$ J.V. Beck, "Transient Determination of Thermal Properties," Nucl. Eng. Des., 3, 373-81 (1966).

16J.V. Beck, "Sequential Estimation of Thermal Parameters," J. Heat Transfer, 99, 314-21 (1977)

${ }^{17}$ R. C. Pfahl, Jr., and B. J. Mitchel, "Simultaneous Measurement of Six Thermal Properties of a Charring Plastic," Int. J. Heat Mass Transfer, 13, $275-86(1970)$.

${ }^{18} \mathrm{~K}$. Ho, A. Jeyarajan, and R. D. Pehlke; unpublished research presented at the Poster Session at the Engineering Foundation Conference on Modeling of Castings and Welding Processes, Rindge, NH, Aug. 1980.

${ }^{19}$ K. Kubo, A. Kasahara, and T. Fukusako, "Thermal Properties of Ceramic Molds (in Jpn.)," J. Jpn. Foundrymen's Soc., 56, 337-43 (1984).

${ }^{20}$ M.J.D. Powell, "An Efficient Method for Finding the Minimum of a Function of Several Variables without Calculating Derivatives," Comput. J., $7,155-62(1965)$.

${ }^{21}$ IMSL Library User's Manual, Edition 9.2, International Mathematical and Statistical Libraries, Inc., Houston, TX, 1984.

${ }^{22}$ E. A. Brandes, Smithel Metals Reference Book, 6th ed. Butterworth, London, U. K., 1983.

${ }^{23}$ M. Ninomiya, Y. Nozaki, Y. Sakaguchi, R. Kurokawa, and M. Sato, "Heat Transfer Properties of Several Kinds of Sand Moulds," Rep. Gov. Ind. Res. Inst., Nagoya, 27, 262-68 (1978).

${ }^{24}$ K. Ho and R. D. Pehlke, "Mechanisms of Heat Transfer at a Metal-Mold Interface," Trans. Am. Foundrymen's Soc., 92, 587-98 (1984). 